\title{
Algebraic properties of the binomial edge ideal of a complete bipartite graph
}

\author{
Peter Schenzel and Sohail Zafar
}

\begin{abstract}
Let $J_{G}$ denote the binomial edge ideal of a connected undirected graph on $n$ vertices. This is the ideal generated by the binomials $x_{i} y_{j}-$ $x_{j} y_{i}, 1 \leq i<j \leq n$, in the polynomial ring $S=K\left[x_{1}, \ldots, x_{n}, y_{1}, \ldots, y_{n}\right]$ where $\{i, j\}$ is an edge of $G$. We study the arithmetic properties of $S / J_{G}$ for $G$, the complete bipartite graph. In particular we compute dimensions, depths, Castelnuovo-Mumford regularities, Hilbert functions and multiplicities of them. As main results we give an explicit description of the modules of deficiencies, the duals of local cohomology modules, and prove the purity of the minimal free resolution of $S / J_{G}$.
\end{abstract}

\section{Introduction}

The main intention of the present paper is the study of the binomial edge ideal of the complete bipartite graph. Let $G$ denote a connected undirected graph on $n$ vertices labeled by $[n]=\{1,2, \ldots, n\}$. For an arbitrary field $K$ let $S=K\left[x_{1}, \ldots, x_{n}, y_{1}, \ldots, y_{n}\right]$ denote the polynomial ring in $2 n$ variables. To the graph $G$ one can associate the binomial edge ideal $J_{G} \subset S$ generated by binomials $x_{i} y_{j}-x_{j} y_{i}, i<j$, such that $\{i, j\}$ is an edge of $G$. This is an extension of the edge ideal (generated by the monomials) as it was studied for instance in [12]. The binomial edge ideals of a graph $G$ might have applications in algebraic statistics (see [6]). By the work of Herzog et al. (see [6]) the

Key Words: binomial edge ideal, complete bipartite graph, pure resolution.

2010 Mathematics Subject Classification: 05E40, 13H10, $13 \mathrm{D} 45$.

Received: February, 2013.

Revised: February, 2013.

Accepted: June, 2013. 
minimal primary decomposition of $J_{G}$ is known. Besides of that not so much is known about the arithmetic properties of $S / J_{G}$. If $G$ denotes the complete graph on $n$ vertices, then $S / J_{G}$ is the coordinate ring of the Segre embedding $\mathbb{P}_{K}^{1} \times \mathbb{P}_{K}^{n}$. This is a variety of minimal degree. Therefore $S / J_{G}$ is a CohenMacaulay ring with a linear resolution. In the paper Ene, Herzog and Hibi (see [4]) they studied Cohen-Macaulayness property for some special classes of graphs. By view of the primary decomposition of $J_{G}$ (see [6]) it follows that $S / J_{G}$ is not so often a Cohen-Macaulay ring. As a certain generalization of the Cohen-Macaulay property the second author has studied approximately Cohen-Macaulay rings (see [13]). In the present paper we investigate the binomial edge ideal of another important class of graphs, namely the complete bipartite graph $G=K_{m, n}$ (see the definitions in Section 3).

As the main result of our investigations we prove (among others) the following results:

Theorem 1.1. With the previous notation let $J_{G} \subset S$ denote the binomial edge ideal associated to the complete bipartite graph $G=K_{m, n}$.

(a) $\operatorname{dim} S / J_{G}=\max \{n+m+1,2 m\}$ and

$$
\operatorname{depth} S / J_{G}= \begin{cases}m+2, & \text { if } n=1 \\ n+2, & \text { if } m \geq n>1\end{cases}
$$

(b) There is an explicit expression of the Hilbert series and the multiplicity equals

$$
e\left(S / J_{G}\right)= \begin{cases}1, & \text { if } m>n+1 \text { or } n=1 \text { and } m>2 \\ 2 m, & \text { otherwise. }\end{cases}
$$

(c) The Castelnuovo-Mumford regularity is $\operatorname{reg} S / J_{G}=2$ and $S / J_{G}$ admits a pure minimal free resolution.

(d) There are at most 5 non-vanishing local cohomology modules $H_{S_{+}}^{i}\left(S / J_{G}\right)$. The modules of deficiencies $\omega^{i}\left(S / J_{G}\right)=\operatorname{Hom}_{K}\left(H_{S_{+}}^{i}\left(S / J_{G}\right), K\right)$ are either Cohen-Macaulay modules or the direct sum of two Cohen-Macaulay modules.

(e) $S / J_{G}$ is a Cohen-Macaulay canonically ring in the sense of [10].

For the details on the modules of deficiencies we refer to Section 4 of the paper. This is - at least for us - the first time in the literature that there is a complete description of the structure of the modules of deficiencies besides of 
sequentially Cohen-Macaulay rings or Buchsbaum rings. Our analysis is based on the primary decomposition of $J_{G}$ as shown in [6].

In Section 2 we start with preliminary and auxiliary results needed in the rest of the paper. In particular we give a short overview on the modules of deficiencies. In Section 3 we study some of the properties of the the binomial edge ideal $J_{G} \subset S$ associated to a complete bipartite graph. In Section 4 we give a complete list of all the modules of deficiencies of the complete bipartite graphs. In the final Section 5 we prove the purity of the minimal free resolution of $S / J_{G}$. This is the heart of our investigations. It gives in a natural way some non-Cohen-Macaulay rings with pure resolutions. We might relate our investigations as a better understanding of general binomial edge ideals.

\section{Preliminaries and auxiliary results}

First of all we will introduce the notation used in the sequel. Moreover we summarize a few auxiliary results that we need.

We denote by $G$ a connected undirected graph on $n$ vertices labeled by $[n]=$ $\{1,2, \ldots, n\}$. For an arbitrary field $K$ let $S=K\left[x_{1}, \ldots, x_{n}, y_{1}, \ldots, y_{n}\right]$ denote the polynomial ring in the $2 n$ variables $x_{1}, \ldots, x_{n}, y_{1}, \ldots, y_{n}$. To the graph $G$ one can associate an ideal $J_{G} \subset S$ generated by all binomials $x_{i} y_{j}-x_{j} y_{i}$ for all $1 \leq i<j \leq n$ such that $\{i, j\}$ is an edge of $G$. This construction was invented by Herzog et al. in [6] and [4]. At first let us recall some of their definitions.

Definition 2.1. Fix the previous notation. For a set $T \subset[n]$ let $\tilde{G}_{T}$ denote the complete graph on the vertex set $T$. Moreover let $G_{[n] \backslash T}$ denote the graph obtained by deleting all vertices of $G$ that belong to $T$.

Let $c=c(T)$ denote the number of connected components of $G_{[n] \backslash T}$. Let $G_{1}, \ldots, G_{c}$ denote the connected components of $G_{[n] \backslash T}$. Then define

$$
P_{T}(G)=\left(\cup_{i \in T}\left\{x_{i}, y_{i}\right\}, J_{\tilde{G}_{1}}, \ldots, J_{\tilde{G}_{C(T)}}\right),
$$

where $\tilde{G}_{i}, i=1, \ldots, c$, denotes the complete graph on the vertex set of the connected component $G_{i}, i=1, \ldots, c$.

The following result is important for the understanding of the binomial edge ideal of $G$.

Lemma 2.2. With the previous notation the following holds:

(a) $P_{T}(G) \subset S$ is a prime ideal of height $n-c+|T|$, where $|T|$ denotes the number of elements of $T$. 
(b) $J_{G}=\cap_{T \subseteq[n]} P_{T}(G)$.

(c) $J_{G} \subset P_{T}(G)$ is a minimal prime if and only if either $T=\emptyset$ or $T \neq \emptyset$ and $c(T \backslash\{i\})<c(T)$ each $i \in T$.

Proof. For the proof we refer to [6].

Therefore $J_{G}$ is the intersection of prime ideals. That is, $S / J_{G}$ is a reduced ring. Moreover, we remark that $J_{G}$ is an ideal generated by quadrics and therefore homogeneous, so that $S / J_{G}$ is a graded ring with natural grading induced by the $\mathbb{N}$-grading of $S$. As a technical tool we shall need the following result.

Proposition 2.3. Let $I \subset S$ denote an ideal. Let $\underline{f}=f_{1}, \ldots, f_{r}$ denote an $S / I$-regular sequence. Then $\underline{f} S \cap I=\underline{f I}$.

Proof. It is easy to see that $\operatorname{Tor}_{1}^{S}(S / \underline{f} S, S / I) \cong \underline{f} S \cap I / \underline{f} I$. Moreover

$$
\operatorname{Tor}_{1}^{S}(S / \underline{f} S, S / I) \cong H_{1}(\underline{f} ; S / I),
$$

where $H_{i}(\underline{f} ; S / I)$ denotes the Koszul homology of $\underline{f}$ with respect to $S / I$. But these homology modules vanish for $i>0$.

Let $M$ denote a finitely generated graded $S$-module. In the sequel we shall use also the local cohomology modules of $M$ with respect to $S_{+}$, denoted by $H^{i}(M), i \in \mathbb{Z}$. Note that they are graded Artinian $S$-modules. We refer to the textbook of Brodmann and Sharp (see [1]) for the basics on it. In particular the Castelnuovo-Mumford regularity reg $M$ of $M$ is defined as

$$
\operatorname{reg}(M):=\max \left\{e\left(H^{i}(M)\right)+i \mid \operatorname{depth}(M) \leq i \leq \operatorname{dim}(M)\right\},
$$

where $e\left(H^{i}(M)\right)$ is the least integer $m$ such that, for all $k>m$, the degree $k$ part of the $i$-th local cohomology module of $\mathrm{M}$ is zero. For our investigations we need the following definition.

Definition 2.4. Let $M$ denote a finitely generated graded $S$-module and $d=\operatorname{dim} M$. For an integer $i \in \mathbb{Z}$ put

$$
\omega^{i}(M)=\operatorname{Ext}_{S}^{2 n-i}(M, S(-2 n))
$$

and call it the $i$-th module of deficiency. Moreover we define $\omega(M)=\omega^{d}(M)$ the canonical module of $M$. We write also $\omega_{2 \times}(M)=\omega(\omega(M))$. These modules have been introduced and studied in [8]. 
Note that by the graded version of Local Duality (see e.g. [1]) there is the natural graded isomorphism $\omega^{i}(M) \cong \operatorname{Hom}_{K}\left(H^{i}(M), K\right)$ for all $i \in \mathbb{Z}$. For a finitely generated graded $S$-module $M$ and an integer $i \in \mathbb{N}$ we set

$$
(\operatorname{Ass} M)_{i}=\{\mathfrak{p} \in \operatorname{Ass} M \mid \operatorname{dim} S / \mathfrak{p}=i\} .
$$

In the following we shall summarize a few properties on the modules of deficiencies.

Proposition 2.5. Let $M$ denote a finitely generated graded $S$-module and $d=\operatorname{dim} M$.

(a) $\operatorname{dim} \omega^{i}(M) \leq i$ and $\operatorname{dim} \omega^{d}(M)=d$.

(b) $\left(\operatorname{Ass} \omega^{i}(M)\right)_{i}=(\operatorname{Ass} M)_{i}$ for all $0 \leq i \leq d$.

(c) $M$ satisfies the Serre condition $S_{2}$ if and only if $\operatorname{dim} \omega^{i}(M) \leq i-2$ for all $0 \leq i<d$.

(d) There is a natural homomorphism $M \rightarrow \omega^{d}\left(\omega^{d}(M)\right)$. It is an isomorphism if and only if $M$ satisfies the Serre condition $S_{2}$.

(e) For a homogeneous ideal $I \subset S$ there is a natural isomorphism $\omega^{d}\left(\omega^{d}(S / I)\right) \cong \operatorname{Hom}_{S}\left(\omega^{d}(S / I), \omega^{d}(S / I)\right), d=\operatorname{dim} S / I$, and it admits the structure of a commutative Noetherian ring, the $S_{2}$-fication of $S / I$.

(f) The natural map $S / I \rightarrow \operatorname{Hom}_{S}\left(\omega^{d}(S / I), \omega^{d}(S / I)\right), d=\operatorname{dim} S / I$, sends the unit element to the identity map. Therefore it is a ring homomorphism.

Proof. The results are shown in [8] and [10]. The proofs in the graded case follow the same line of arguments.

A decreasing sequence $\left\{M_{i}\right\}_{0 \leq i \leq d}$ of a $d$-dimensional $S$-module $M$ is called dimension filtration of $M$, if $M_{i} / M_{i-1}$ is either zero or of dimension $i$ for all $i=0, \ldots, d$, where $M_{-1}=0$. It was shown (see [9]) that the dimension filtration exists and is uniquely determined.

Definition 2.6. An $S$-module $M$ is called sequentially Cohen-Macaulay if the dimension filtration $\left\{M_{i}\right\}_{0 \leq i \leq d}$ has the property that $M_{i} / M_{i-1}$ is either zero or an $i$-dimensional Cohen-Macaulay module for all $i=0, \ldots, d$, (see [9]). Note that in [9] this notion was originally called Cohen-Macaulay filtered.

Note that a sequentially Cohen-Macaulay $S$-module $M$ with $\operatorname{depth} M \geq$ $\operatorname{dim} M-1$ was studied by Gôto (see [5]) under the name approximately CohenMacaulay. For our purposes here we need the following characterization of sequentially Cohen-Macaulay modules. 
Theorem 2.7. Let $M$ be a finitely generated graded $S$-module with $d=\operatorname{dim} M$. Then the following conditions are equivalent:

(i) $M$ is a sequentially Cohen-Macaulay.

(ii) For all $0 \leq i<d$ the module of deficiency $\omega^{i}(M)$ is either zero or an $i$-dimensional Cohen-Macaulay module.

(iii) For all $0 \leq i \leq d$ the modules $\omega^{i}(M)$ are either zero or $i$-dimensional Cohen-Macaulay modules.

Proof. In the case of a local ring admitting a dualizing complex this result was shown in [9, Theorem 5.5]. Similar arguments work also in the case of a finitely generated graded $S$-module $M$. Note that the equivalence of (i) and (iii) was announced (without proof) in [11].

\section{Complete bipartite graphs}

A bipartite graph is a graph whose vertices can be divided into two disjoint sets $V_{1}$ and $V_{2}$ such every edge of $G$ connects a vertex in $V_{1}$ to one in $V_{2}$. Now the complete bipartite graph is a bipartite graph $G$ such that for any two vertices, $v_{1} \in V_{1}$ and $v_{2} \in V_{2}, v_{1} v_{2}$ is an edge in $G$. If $\left|V_{1}\right|=n$ and $\left|V_{2}\right|=m$ then it is usually denoted by $K_{n, m}$. To simplifying notations we denote it often by $G$.

Definition 3.1. For a sequence of integers $1 \leq i_{1}<i_{2}<\ldots<i_{k} \leq n+m$ let $I\left(i_{1}, i_{2}, \ldots, i_{k}\right)$ denote the ideal generated by the $2 \times 2$ minors of the matrix

$$
\left(\begin{array}{cccc}
x_{i_{1}} & x_{i_{2}} & \cdots & x_{i_{k}} \\
y_{i_{1}} & y_{i_{2}} & \cdots & y_{i_{k}}
\end{array}\right) .
$$

Note that $I\left(i_{1}, i_{2}, \ldots, i_{k}\right)$ is the ideal of the complete graph on the vertex set $\left\{i_{1}, i_{2}, \ldots, i_{k}\right\}$.

Let $J_{G}$ be the binomial edge ideal of complete bipartite graph on $[n+m]$ vertices and $J_{\tilde{G}}$ be the binomial edge ideal of complete graph on $[n+m]$ vertices. We begin with a lemma concerning the dimension of $S / J_{G}$.

Lemma 3.2. Let $G=K_{m, n}, m \geq n$, denote the complete bipartite graph. Let $\tilde{G}$ denote the complete graph on $[n+m]$. Let $A_{n}=\left(x_{1}, \ldots, x_{n}, y_{1}, \ldots, y_{n}\right)$ for $n \geq 1$ and $B_{m}=\left(x_{n+1}, \ldots, x_{n+m}, y_{n+1}, \ldots, y_{n+m}\right)$ for $n \geq 2$ and $B_{m}=S$ for $n=1$.

(a) $J_{G}=J_{\tilde{G}} \cap A_{n} \cap B_{m}$ is the minimal primary decomposition of $J_{G}$. 
(b) $\operatorname{dim} S / J_{G}=\max \{n+m+1,2 m\}$.

(c) $\left(J_{\tilde{G}} \cap A_{n}, J_{\tilde{G}} \cap B_{m}\right)=J_{\tilde{G}}$.

Proof. We start with the proof of (a). We use the statement proved in Lemma 2.2. At first consider the case $m>n=1$. By view of Lemma 2.2 we have to find all $\emptyset \neq T \subseteq[1+m]$ such that $c(T \backslash\{i\})<c(T)$. Clearly $T_{0}=\{1\}$ satisfy the condition because $c\left(T_{0}\right)=m>1$. Let $T$ denote $T \subset[1+m]$ a subset different of $T_{0}$. Then If $1 \in T$ then $c(T)=m+1-|T|$ and $c(T \backslash\{i\})=m+2-|T|$ for $i \neq 1$ and if $1 \notin T$ then $c(T)=1$ and $c(T \backslash\{i\})=1$ for all $i \in T$. Hence we have the above primary decomposition.

Now consider the case of $m \geq n \geq 2$. As above we have to find all $\emptyset \neq T \subseteq[n+m]$ such that $c(T \backslash\{i\})<c(T)$ for all $i \in T$. $T_{1}=\{1,2, \ldots, n\}$ satisfy the above condition because $c(T)=m$ and $c(T \backslash\{i\})=1$ for all $i \in T$. Similarly $T_{2}=\{n+1, n+2, \ldots, n+m\}$ also satisfies the above condition.

Our claim is that no other $T \subseteq[n+m]$ satisfies this condition. If $T_{1} \nsubseteq T$ and $T_{2} \not \subseteq T$ then $c(T)=1$ so in this case $T$ does not satisfy the above condition. Now suppose that $T_{1} \subsetneq T$ then $c(T)=m-\left|T \backslash T_{1}\right|$ and $c(T \backslash\{i\})=$ $m+1-\left|T \backslash T_{1}\right|$ if $i \in T \backslash T_{1}$. The same argument works if $T_{2} \subsetneq T$. Hence we have $J_{G}=J_{\tilde{G}} \cap A_{n} \cap B_{m}$.

Then the statement on the dimension in (b) is a consequence of the reduced primary decomposition shown in (a). To this end recall that $\operatorname{dim} S / A_{n}=$ $2 m, \operatorname{dim} S / B_{m}=2 n$ and $\operatorname{dim} S / J_{\tilde{G}}=n+m+1$.

For the proof of (c) we use the notation of the Definition 3.1. Then it follows that

$$
J_{\tilde{G}} \cap A_{n}=\left(I(1, \ldots, n, n+i), i=1, \ldots, m, I(n+1, \ldots, n+m) \cap A_{n}\right) .
$$

Now $A_{n}$ consists of an $S / I(n+1, \ldots, n+m)$-regular sequence and

$$
I(n+1, \ldots, n+m) \cap A_{n}=A_{n} I(n+1, \ldots, n+m)
$$

by Proposition 2.3. Therefore we get

$$
J_{\tilde{G}} \cap A_{n}=\left(I(1, \ldots, n, n+i), i=1, \ldots, m, A_{n} I(n+1, \ldots, n+m)\right)
$$

and similarly

$$
J_{\tilde{G}} \cap B_{m}=\left(I(j, n+1, \ldots, n+m), j=1, \ldots, n, B_{m} I(1, \ldots, n)\right) .
$$

But this clearly implies that $\left(J_{\tilde{G}} \cap A_{n}, J_{\tilde{G}} \cap B_{m}\right)=J_{\tilde{G}}$ which proves the statement in (c).

For the further computations we use the previous Lemma 3.2. In particular we use three exact sequences shown in the next statement. 
Corollary 3.3. With the previous notation we have the following three exact sequences.

(1) $0 \rightarrow S / J_{G} \rightarrow S / J_{\tilde{G}} \cap A_{n} \oplus S / J_{\tilde{G}} \cap B_{m} \rightarrow S / J_{\tilde{G}} \rightarrow 0$.

(2) $0 \rightarrow S / J_{\tilde{G}} \cap A_{n} \rightarrow S / J_{\tilde{G}} \oplus S / A_{n} \rightarrow S /\left(J_{\tilde{G}}, A_{n}\right) \rightarrow 0$.

(3) $0 \rightarrow S / J_{\tilde{G}} \cap B_{m} \rightarrow S / J_{\tilde{G}} \oplus S / B_{m} \rightarrow S /\left(J_{\tilde{G}}, B_{m}\right) \rightarrow 0$.

Proof. The proof is an easy consequence of the primary decomposition as shown in Lemma 3.2. We omit the details.

Note that in case of $n=1$ we have $B_{m}=S$ therefore it is enough to consider the exact sequence (2) as (1) and (3) gives no information.

Corollary 3.4. With the previous notation we have that

$$
\operatorname{depth} S / J_{G}= \begin{cases}m+2, & \text { if } n=1 ; \\ n+2, & \text { if } m \geq n>1\end{cases}
$$

and $\operatorname{reg} S / J_{G} \leq 2$.

Proof. The statement is an easy consequence of the short exact sequences shown in Corollary 3.3. To this end note that $S / J_{\tilde{G}}, S /\left(J_{\tilde{G}}, A_{n}\right)$ and $S /\left(J_{\tilde{G}}, B_{m}\right)$ are Cohen-Macaulay rings of dimension $n+m+1, m+1$ and $n+1$ respectively. Moreover $\operatorname{reg} S / J_{\tilde{G}}=\operatorname{reg} S /\left(J_{\tilde{G}}, A_{n}\right)=\operatorname{reg} S /\left(J_{\tilde{G}}, B_{m}\right)=1$. By using the exact sequences it provides the statement on the regularity. For the behaviour of the depth respectively the regularity in short exact sequences see [2, Proposition 1.2.9] respectively [3, Corollary 20.19].

\section{The modules of deficiency}

The goal of this section is to describe all the local cohomology modules $H^{i}\left(S / J_{G}\right)$ of the binomial edge ideal of a complete bipartite graph $G$. We do this by describing their Matlis duals which by Local Duality are the modules of deficiencies. Moreover, for a homogeneous ideal $J \subset S$ let $H(S / J, t)$ denote the Hilbert series, i.e. $H(S / J, t)=\sum_{i \geq 0}\left(\operatorname{dim}_{K}[S / J]_{i}\right) t^{i}$.

We start our investigations with the so-called star graph. That is complete bipartite graph $K_{m, n}$ with $n=1$. For $m \leq 2$ the ideal $J_{G}$ is a complete intersection generated by one respectively two quadrics so let us assume that $m>2$.

Theorem 4.1. Let $G$ denote the star graph $K_{m, 1}$. Then the binomial edge ideal $J_{G} \subset S$ has the following properties: 
(a) $\operatorname{reg} S / J_{G}=2$.

(b) $\omega^{i}\left(S / J_{G}\right)=0$ if and only if $i \notin\{m+2,2 m\}$.

(c) $\omega^{2 m}\left(S / J_{G}\right) \cong S / A_{1}(-2 m)$

(d) $\omega^{m+2}\left(S / J_{G}\right)$ is a $(m+2)$-dimensional Cohen-Macaulay module and there is an isomorphism $\omega^{m+2}\left(\omega^{m+2}\left(S / J_{G}\right)\right) \cong\left(J_{\tilde{G}}, A_{1}\right) / J_{\tilde{G}}$.

Proof. We use the short exact sequence of Corollary 3.3 (2). It induces a short exact sequence

$$
0 \rightarrow H^{m+1}\left(S /\left(J_{\tilde{G}}, A_{1}\right)\right) \rightarrow H^{m+2}\left(S / J_{G}\right) \rightarrow H^{m+2}\left(S / J_{\tilde{G}}\right) \rightarrow 0
$$

and an isomorphism $H^{2 m}\left(S / J_{G}\right) \cong H^{2 m}\left(S / A_{1}\right)$. Moreover the CohenMacaulayness of $S / J_{\tilde{G}}, S / A_{1}$ and $S /\left(J_{\tilde{G}}, A_{1}\right)$ of dimensions $m+2,2 m$ and $m+1$ respectively imply that $H^{i}\left(S / J_{G}\right)=0$ if $i \notin\{m+2,2 m\}$.

The short exact sequence on local cohomology induces the following exact sequence

$$
0 \rightarrow \omega^{m+2}\left(S / J_{\tilde{G}}\right) \rightarrow \omega^{m+2}\left(S / J_{G}\right) \rightarrow \omega^{m+1}\left(S /\left(J_{\tilde{G}}, A_{1}\right)\right) \rightarrow 0
$$

by Local Duality. Now we apply again local cohomology and take into account that both $\omega^{m+2}\left(S / J_{\tilde{G}}\right)$ and $\omega^{m+1}\left(S /\left(J_{\tilde{G}}, A_{1}\right)\right)$ are Cohen-Macaulay modules of dimension $m+2$ and $m+1$ respectively. Then $\operatorname{depth} \omega^{m+2}\left(S / J_{G}\right) \geq m+1$. By applying local cohomology and dualizing again it induces the following exact sequence

$0 \rightarrow \omega^{m+2}\left(\omega^{m+2}\left(S / J_{G}\right)\right) \rightarrow S / J_{\tilde{G}} \stackrel{f}{\rightarrow} S /\left(J_{\tilde{G}}, A_{1}\right) \rightarrow \omega^{m+1}\left(\omega^{m+2}\left(S / J_{G}\right)\right) \rightarrow 0$.

The homomorphism $f$ is induced by the commutative diagram

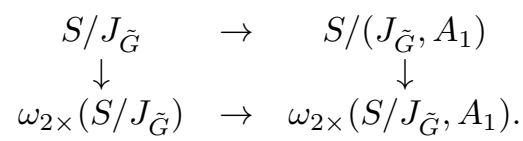

Note that the vertical maps are isomorphisms (see Proposition 2.5). Since the upper horizontal map is surjective the lower horizontal map is surjective too. Therefore $\omega^{m+1}\left(\omega^{m+2}\left(S / J_{G}\right)\right)=0$. That is $\operatorname{depth} \omega^{m+2}\left(S / J_{G}\right)=m+2$ and it is a Cohen-Macaulay module. Moreover $\omega^{m+2}\left(\omega^{m+2}\left(S / J_{G}\right)\right) \cong\left(J_{\tilde{G}}, A_{1}\right) / J_{\tilde{G}}$. This finally proves the statements in (b), (c) and (d).

It is well known that $\operatorname{reg} S / J_{G}=\operatorname{reg} S /\left(J_{\tilde{G}}, A_{1}\right)=1$ and $\operatorname{reg} S / A_{1}=0$. Then an inspection with the short exact sequence of Corollary 3.3 shows that $\operatorname{reg} S / J_{G}=2$. 
In the next result we will consider the modules of deficiencies of the complete bipartite graph $G=K_{m, n}, n \geq 2$.

Theorem 4.2. Let $m \geq n>1$ and assume that the pair $(m, n)$ is different from $(n+1, n)$ and $(2 n-2, n)$. Then

(a) $\operatorname{reg} S / J_{G}=2$.

(b) $\omega^{i}\left(S / J_{G}\right)=0$ if and only if $i \notin\{n+2, m+2,2 n, m+n+1,2 m\}$ and there are the following isomorphisms and integers

\begin{tabular}{c|ccc}
$i$ & $\omega^{i}\left(S / J_{G}\right)$ & $\operatorname{depth} \omega^{i}\left(S / J_{G}\right)$ & $\operatorname{dim} \omega^{i}\left(S / J_{G}\right)$ \\
\hline$n+2$ & $\omega^{n+1}\left(S /\left(J_{\tilde{G}}, B_{m}\right)\right)$ & $n+1$ & $n+1$ \\
$m+2$ & $\omega^{m+1}\left(S /\left(J_{\tilde{G}}, A_{n}\right)\right)$ & $m+1$ & $m+1$ \\
$2 n$ & $S / B_{m}(-2 n)$ & $2 n$ & $2 n$ \\
$n+m+1$ & $\omega^{m+n+1}\left(S / J_{\tilde{G}}\right)$ & $n+m+1$ & $n+m+1$ \\
$2 m$ & $S / A_{n}(-2 m)$ & $2 m$ & $2 m$
\end{tabular}

Proof. Under the assumption of $n+1<m<2 n-2$ it follows that $2 m>$ $m+n+1>2 n>m+2>n+2$. Then the short exact sequences (see Corollary 3.3) induce the following isomorphisms:

(1) $H^{n+2}\left(S / J_{G}\right) \cong H^{n+2}\left(S / J_{\tilde{G}} \cap B_{m}\right) \cong H^{n+1}\left(S /\left(J_{\tilde{G}}, B_{m}\right)\right)$,

(2) $H^{m+2}\left(S / J_{G}\right) \cong H^{m+2}\left(S / J_{\tilde{G}} \cap A_{n}\right) \cong H^{m+1}\left(S /\left(J_{\tilde{G}}, A_{n}\right)\right)$,

(3) $H^{2 n}\left(S / J_{G}\right) \cong H^{2 n}\left(S / B_{m}\right)$ and

(4) $H^{2 m}\left(S / J_{G}\right) \cong H^{2 m}\left(S / A_{n}\right)$.

Moreover there is the following short exact sequence

$$
\begin{aligned}
0 \rightarrow H^{m+n+1}\left(S / J_{G}\right) \rightarrow & H^{m+n+1}\left(S / J_{\tilde{G}} \cap A_{n}\right) \oplus H^{m+n+1}\left(S / J_{\tilde{G}} \cap B_{m}\right) \rightarrow \\
& \rightarrow H^{m+n+1}\left(S / J_{\tilde{G}}\right) \rightarrow 0
\end{aligned}
$$

and $H^{i}\left(S / J_{G}\right)=0$ if $i \notin\{n+2, m+2,2 n, m+n+1,2 m\}$.

Because of the short exact sequences in Corollary 3.3 there are isomorphisms

$$
H^{m+n+1}\left(S / J_{\tilde{G}} \cap B_{m}\right) \cong H^{m+n+1}\left(S / J_{\tilde{G}}\right) \cong H^{m+n+1}\left(S / J_{\tilde{G}} \cap A_{n}\right) .
$$

So by Local Duality we get a short exact sequence

$$
0 \rightarrow \omega\left(S / J_{\tilde{G}}\right) \rightarrow \omega\left(S / J_{\tilde{G}}\right) \oplus \omega\left(S / J_{\tilde{G}}\right) \rightarrow \omega^{m+n+1}\left(S / J_{G}\right) \rightarrow 0
$$


This implies depth $\omega\left(S / J_{G}\right) \geq n+m$. Moreover by applying local cohomology and again the Local Duality we get the following commutative diagram with exact rows

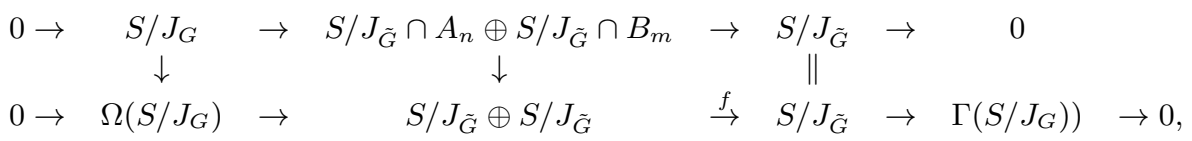

where

$\Omega\left(S / J_{G}\right)=\omega^{m+n+1}\left(\omega^{m+n+1}\left(S / J_{G}\right)\right)$ and $\Gamma\left(S / J_{G}\right)=\omega^{m+n}\left(\omega^{m+n+1}\left(S / J_{G}\right)\right)$.

Now we show that $\left.\Gamma\left(S / J_{G}\right)\right)=0$. This follows since $f$ is easily seen to be surjective. That is, $\omega^{m+n+1}\left(S / J_{G}\right)$ is a $(m+n+1)$-dimensional Cohen-Macaulay module. Moreover $f$ is a split homomorphism and therefore $\Omega\left(S / J_{G}\right) \simeq S / J_{\tilde{G}}$ By duality this implies that $\omega^{m+n+1}\left(S / J_{G}\right) \cong \omega\left(S / J_{\tilde{G}}\right)$. This completes the proof of the statements in (b). By similar arguments the other cases for $(m, n)$ different of $(n+1, n)$ and $(2 n-2, n)$ can be proved. We omit the details. Clearly $\operatorname{reg} S / J_{G}=2$ as follows by (b).

As a next sample of our considerations let us consider the case of the complete bipartite graph $K_{m, n}$ with $(m, n)=(n+1, n)$.

Theorem 4.3. Let $m=n+1$ and $n>3$. Then:

(a) $\operatorname{reg} S / J_{G}=2$.

(b) $\omega^{i}\left(S / J_{G}\right)=0$ if and only if $i \notin\{n+2, n+3,2 n, 2 n+2\}$ and there are the following isomorphisms and integers

\begin{tabular}{c|ccc}
$i$ & $\omega^{i}\left(S / J_{G}\right)$ & $\operatorname{depth} \omega^{i}\left(S / J_{G}\right)$ & $\operatorname{dim} \omega^{i}\left(S / J_{G}\right)$ \\
\hline$n+2$ & $\omega^{n+1}\left(S /\left(J_{\tilde{G}}, B_{n+1}\right)\right)$ & $n+1$ & $n+1$ \\
$n+3$ & $\omega^{n+2}\left(S /\left(J_{\tilde{G}}, A_{n}\right)\right)$ & $n+2$ & $n+2$ \\
$2 n$ & $S / B_{n+1}(-2 n)$ & $2 n$ & $2 n$ \\
$2 n+2$ & $\omega\left(S / J_{\tilde{G}}\right) \oplus S / A_{n}(-2 n-2)$ & $2 n+2$ & $2 n+2$.
\end{tabular}

Proof. By applying the local cohomology functors $H^{\cdot}(-)$ to the exact sequence (2) in Corollary 3.3 we get the following:

(1) $H^{n+3}\left(S / J_{\tilde{G}} \cap A_{n}\right) \cong H^{n+2}\left(S /\left(J_{\tilde{G}}, A_{n}\right)\right)$,

(2) $H^{2 n+2}\left(S / J_{\tilde{G}} \cap A_{n}\right) \cong H^{2 n+2}\left(S / J_{\tilde{G}}\right) \oplus H^{2 n+2}\left(S / A_{n}\right)$ and

(3) $H^{i}\left(S / J_{\tilde{G}} \cap A_{n}\right)=0$ for $i \neq n+2,2 n+2$.

Similarly, if we apply $H^{*}(-)$ to the exact sequence (3) in Corollary 3.3 we get 
(4) $H^{n+2}\left(S / J_{\tilde{G}} \cap B_{n+1}\right) \cong H^{n+1}\left(S /\left(J_{\tilde{G}}, B_{n+1}\right)\right)$.

(5) $H^{2 n}\left(S / J_{\tilde{G}} \cap B_{n+1}\right) \cong H^{2 n}\left(S / B_{n+1}\right)$.

(6) $H^{2 n+2}\left(S / J_{\tilde{G}} \cap B_{n+1}\right) \cong H^{2 n+2}\left(S / J_{\tilde{G}}\right)$.

(7) $H^{i}\left(S / J_{\tilde{G}} \cap B_{n+1}\right)=0$ for $i \neq n+2,2 n, 2 n+2$.

With these results in mind the short exact sequence (1) of Corollary 3.3 provides (by applying the local cohomology functor) the vanishing $H^{i}\left(S / J_{G}\right)=0$ for all $i \neq n+2, n+3,2 n, 2 n+2$. Moreover it induces isomorphisms

$$
H^{n+2}\left(S / J_{G}\right) \cong H^{n+1}\left(S /\left(J_{\tilde{G}}, B_{n+1}\right)\right) \text { and } H^{2 n}\left(S / J_{G}\right) \cong H^{2 n}\left(S / B_{n+1}\right)
$$

and as $n>3$ so $2 n>n+3$ the isomorphism $H^{n+3}\left(S / J_{G}\right) \cong H^{n+2}\left(S /\left(J_{\tilde{G}}, A_{n}\right)\right)$. Moreover we obtain the following short exact sequence

$$
\begin{gathered}
0 \rightarrow H^{2 n+2}\left(S / J_{G}\right) \rightarrow H^{2 n+2}\left(S / J_{\tilde{G}}\right) \oplus H^{2 n+2}\left(S / A_{n}\right) \oplus H^{2 n+2}\left(S / J_{\tilde{G}}\right) \rightarrow \\
\rightarrow H^{2 n+2}\left(S / J_{\tilde{G}}\right) \rightarrow 0 .
\end{gathered}
$$

By Local Duality this proves the first three rows in the table of statement (b). By Local Duality we get also the the following short exact sequence

$$
0 \rightarrow \omega\left(S / J_{\tilde{G}}\right) \rightarrow \omega\left(S / J_{\tilde{G}}\right) \oplus \omega\left(S / A_{n}\right) \oplus \omega\left(S / J_{\tilde{G}}\right) \rightarrow \omega\left(S / J_{G}\right) \rightarrow 0 .
$$

Note that we may write $\omega$ instead of $\omega^{2 n+2}$ because all modules above are canonical modules. First of all the short exact sequence provides that $\operatorname{depth} \omega\left(S / J_{G}\right) \geq 2 n+1$. By applying local cohomology and dualizing again we get the following exact sequence

$$
0 \rightarrow \omega_{2 \times}\left(S / J_{G}\right) \rightarrow S / J_{\tilde{G}} \oplus S / A_{n} \oplus S / J_{\tilde{G}} \stackrel{f}{\rightarrow} S / J_{\tilde{G}} \rightarrow \omega^{2 n+1}\left(\omega\left(S / J_{G}\right)\right) \rightarrow 0 .
$$

As in the proof of Theorem 4.1 we see that $f$ is surjective. Therefore $\omega^{2 n+1}\left(\omega\left(S / J_{G}\right)\right)=0$ and $\operatorname{depth} \omega\left(S / J_{G}\right)=2 n+2$. Whence $\omega\left(S / J_{G}\right)$ is a $(2 n+2)$-dimensional Cohen-Macaulay module. Then $f$ is a split surjection and $\omega_{2 \times}\left(S / J_{G}\right) \cong S / J_{\tilde{G}} \oplus S / A_{n}$. This implies the isomorphism $\omega\left(S / J_{G}\right) \cong$ $\omega\left(S / J_{\tilde{G}}\right) \oplus \omega\left(S / A_{n}\right)$ and this finishes the proof of (b). Clearly reg $S / J_{G}=$ 2.

Theorem 4.4. Let $m>3$ and $n=2$. Then:

(a) $\operatorname{reg} S / J_{G}=2$. 
(b) $\omega^{i}\left(S / J_{G}\right)=0$ if and only if $i \notin\{4, m+2, m+3,2 m\}$ and there are the following isomorphisms and integers

\begin{tabular}{c|ccc}
$i$ & $\omega^{i}\left(S / J_{G}\right)$ & $\operatorname{depth} \omega^{i}\left(S / J_{G}\right)$ & $\operatorname{dim} \omega^{i}\left(S / J_{G}\right)$ \\
\hline 4 & $\omega^{4}\left(\left(J_{\tilde{G}}, B_{m}\right) / B_{m}\right)$ & 4 & 4 \\
$m+2$ & $\omega^{m+1}\left(S /\left(J_{\tilde{G}}, A_{2}\right)\right)$ & $m+1$ & $m+1$ \\
$m+3$ & $\omega\left(S / J_{\tilde{G}}\right)$ & $m+3$ & $m+3$ \\
$2 m$ & $S / A_{2}(-2 m)$ & $2 m$ & $2 m$.
\end{tabular}

Moreover there is an isomorphism $\omega^{4}\left(\omega^{4}\left(S / J_{G}\right) \cong\left(J_{\tilde{G}}, B_{m}\right) / B_{m}\right.$.

Proof. By applying the local cohomology functors $H^{\cdot}(-)$ to the exact sequence (2) in Corollary 3.3 we get the following:

(1) $H^{m+2}\left(S / J_{\tilde{G}} \cap A_{2}\right) \cong H^{m+1}\left(S /\left(J_{\tilde{G}}, A_{2}\right)\right)$,

(2) $H^{m+3}\left(S / J_{\tilde{G}} \cap A_{2}\right) \cong H^{m+3}\left(S / J_{\tilde{G}}\right)$,

(3) $H^{2 m}\left(S / J_{\tilde{G}} \cap A_{2}\right) \cong H^{2 m}\left(S / A_{2}\right)$ and

(4) $H^{i}\left(S / J_{\tilde{G}} \cap A_{2}\right)=0$ for $i \neq m+2, m+3,2 m$.

Similarly, if we apply $H^{\cdot}(-)$ to the exact sequence (3) in Corollary 3.3 we get the isomorphism $H^{m+3}\left(S / J_{\tilde{G}} \cap B_{m}\right) \cong H^{m+3}\left(S / J_{\tilde{G}}\right)$ and the exact sequence

$$
0 \rightarrow H^{3}\left(S /\left(J_{\tilde{G}}, B_{m}\right)\right) \rightarrow H^{4}\left(S / J_{\tilde{G}} \cap B_{m}\right) \rightarrow H^{4}\left(S / B_{m}\right) \rightarrow 0 .
$$

The short exact sequence on local cohomology induces the following exact sequence

$$
0 \rightarrow \omega^{4}\left(S / B_{m}\right) \rightarrow \omega^{4}\left(S / J_{\tilde{G}} \cap B_{m}\right) \rightarrow \omega^{3}\left(S /\left(J_{\tilde{G}}, B_{m}\right)\right) \rightarrow 0
$$

by Local Duality. Now we apply again local cohomology and taking into account that both $\omega^{4}\left(S / B_{m}\right)$ and $\omega^{3}\left(S /\left(J_{\tilde{G}}, B_{m}\right)\right)$ are Cohen-Macaulay modules of dimension 4 and 3 respectively. Then $\left.\operatorname{depth} \omega^{4}\left(S / J_{\tilde{G}} \cap B_{m}\right)\right) \geq 3$. By applying local cohomology and dualizing again it induces the following exact sequence

$0 \rightarrow \omega^{4}\left(\omega^{4}\left(S / J_{\tilde{G}} \cap B_{m}\right)\right) \rightarrow S / B_{m} \stackrel{f}{\rightarrow} S /\left(J_{\tilde{G}}, B_{m}\right) \rightarrow \omega^{3}\left(\omega^{4}\left(S / J_{\tilde{G}} \cap B_{m}\right)\right) \rightarrow 0$.

Now the homomorphism $f$ is an epimorphism. $\omega^{3}\left(\omega^{4}\left(S / J_{\tilde{G}} \cap B_{m}\right)\right)=0$. That is depth $\omega^{4}\left(S / J_{\tilde{G}} \cap B_{m}\right)=4$ and it is a Cohen-Macaulay module. Moreover $\omega^{4}\left(\omega^{4}\left(S / J_{\tilde{G}} \cap B_{m}\right)\right) \cong\left(J_{\tilde{G}}, B_{m}\right) / B_{m}$. With these results in mind the short exact sequence (1) of Corollary 3.3 provides (by applying the local cohomology 
functor) the vanishing $H^{i}\left(S / J_{G}\right)=0$ for all $i \neq 4, m+2, m+3,2 m$. Moreover it induces isomorphisms

$$
H^{4}\left(S / J_{G}\right) \cong H^{4}\left(S / J_{\tilde{G}} \cap B_{m}\right), H^{m+2}\left(S / J_{G}\right) \cong H^{m+1}\left(S /\left(J_{\tilde{G}}, A_{2}\right)\right)
$$

and $H^{2 m}\left(S / J_{G}\right) \cong H^{2 m}\left(S / A_{2}\right)$. Moreover we obtain the following short exact sequence

$$
0 \rightarrow H^{m+3}\left(S / J_{G}\right) \rightarrow H^{m+3}\left(S / J_{\tilde{G}}\right) \oplus H^{m+3}\left(S / J_{\tilde{G}}\right) \rightarrow H^{m+3}\left(S / J_{\tilde{G}}\right) \rightarrow 0 .
$$

This implies the isomorphism $\omega^{m+3}\left(S / J_{G}\right) \cong \omega\left(S / J_{\tilde{G}}\right)$.

As a final step we shall consider the case of the complete bipartite graph $K_{m, n}$ with $2 n=m+2$. In all of the previous examples we have the phenomenon that $\omega^{i}\left(S / J_{G}\right)$ is either zero or a Cohen-Macaulay module with $i-1 \leq \operatorname{dim} \omega^{i}\left(S / J_{G}\right) \leq i$ for all $i \in \mathbb{Z}$. and the canonical module $\omega\left(S / J_{G}\right)=$ $\omega^{d}\left(S / J_{G}\right), d=\operatorname{dim} S / J_{G}$, is a $d$-dimensional Cohen-Macaulay module. For $2 n=m+2$ this is no longer true.

Theorem 4.5. Let $m+2=2 n$ and $m>n+1$. Then:

(a) $\operatorname{reg} S / J_{G}=2$.

(b) $\omega^{i}\left(S / J_{G}\right)=0$ if and only if $i \notin\{n+2, m+2=2 n, m+n+1,2 m\}$ and there are the following isomorphisms and integers

\begin{tabular}{c|ccc}
$i$ & $\omega^{i}\left(S / J_{G}\right)$ & $\operatorname{depth} \omega^{i}\left(S / J_{G}\right)$ & $\operatorname{dim} \omega^{i}\left(S / J_{G}\right)$ \\
\hline$n+2$ & $\omega^{n+1}\left(S /\left(J_{\tilde{G}}, B_{m}\right)\right)$ & $n+1$ & $n+1$ \\
$m+2$ & $\omega^{m+1}\left(S /\left(J_{\tilde{G}}, A_{n}\right)\right) \oplus S / B_{m}(-2 n)$ & $m+1$ & $m+2$ \\
$m+n+1$ & $\omega\left(S / J_{\tilde{G}}\right)$ & $m+n+1$ & $m+n+1$ \\
$2 m$ & $S / A_{n}(-2 m)$ & $2 m$ & $2 m$.
\end{tabular}

Proof. It is easily seen that $n+2<2 n=m+2<m+n+1<2 m$. Then the short exact sequences of Corollary 3.3 provide that $H^{i}\left(S / J_{G}\right)=0$ for all $i \neq n+2, m+2=2 n, m+n+1,2 m$. Moreover, it induces the following isomorphisms

(1) $H^{n+2}\left(S / J_{G}\right) \cong H^{n+1}\left(S /\left(J_{\tilde{G}}, B_{m}\right)\right)$,

(2) $H^{m+2}\left(S / J_{G}\right) \cong H^{m+1}\left(S /\left(J_{\tilde{G}}, A_{n}\right) \oplus H^{m+2}\left(S / B_{m}\right)\right.$,

(3) $H^{m+n+1}\left(S / J_{G}\right) \cong H^{m+n+1}\left(S / J_{\tilde{G}}\right)$ and

(4) $H^{2 m}\left(S / J_{G}\right) \cong H^{2 m}\left(S / A_{n}\right)$.

This easily yields the statements in (a) and (b). 
The difference of the case handled in Theorem 4.5 is the fact the $\omega^{m+2}\left(S / J_{G}\right)$ is not a Cohen-Macaulay module. It is the direct sum of two Cohen-Macaulay modules of dimensions $m+2$ and $m+1$ respectively. In [10] a finitely generated $S$-module $M$ is called canonically Cohen-Macaulay module whenever $\omega(M)$ is a Cohen-Macaulay module. Note that if $M$ is a Cohen-Macaulay module, then it is also a Cohen-Macaulay canonical module. The converse is not true.

Now we prove some corollaries about the Cohen-Macaulayness and related properties.

Corollary 4.6. Let $J_{G} \subset S$ denote the binomial edge ideal of a complete bipartite graph.

(a) $S / J_{G}$ is a Cohen-Macaulay canonical ring and depth $\omega^{i}\left(S / J_{G}\right) \geq i-1$ for all $\operatorname{depth} S / J_{G} \leq i \leq \operatorname{dim} S / J_{G}$. Moreover $S / J_{G}$ is a Cohen-Macaulay ring if and only if $(m, n) \in\{(2,1)(1,1)\}$.

(b) $S / J_{G}$ is sequentially Cohen-Macaulay and not Cohen-Macaulay if and only if $n=1$ and $m>2$ or $n=m=2$.

Proof. By view of Theorems 4.1, 4.2, 4.3, 4.4 and 4.5 we get the statements on the Cohen-Macaulayness of $\omega\left(S / J_{G}\right)$ and the estimates of of the depth of $\omega^{i}\left(S / J_{G}\right)$ for all possible bipartite graphs $G$. By Lemma 3.2 and Corollary 3.4 the claim on the Cohen-Macaulayness of $S / J_{G}$ is easily seen. Similar arguments work for the sequentially Cohen-Macaulay property as it is easily seen by the definition.

\section{On the purity of the free resolution}

In the following let $J_{r} \subset S, r \leq m+n$, denote the binomial edge ideal corresponding to the complete graph on $r$ vertices. As a technical tool for our further investigations we need the following Lemma.

Lemma 5.1. (a) Let $M$ denote a finitely generated graded $S$-module. Let $\underline{f}=f_{1}, \ldots, f_{l}$ denote an $M$-regular sequence of forms of degree 1 . Then

$$
\operatorname{Tor}_{i}^{S}(K, M / \underline{f} M) \cong \oplus_{j=0}^{l} \operatorname{Tor}_{i-j}^{S}(K, M)^{\left(\begin{array}{l}
l \\
j
\end{array}\right)}(-j) .
$$

(b) $\operatorname{Tor}_{i}^{S}\left(K, S / J_{r}\right) \cong K^{b_{i}(r)}(-i-1)$ for $i=1, \ldots, r-1$, where $b_{i}(r)=i\left(\begin{array}{c}r \\ i+1\end{array}\right)$.

Proof. For the proof of the statement in (a) let $l=1$ and $f=f_{1}$. Then the short exact sequence $0 \rightarrow M(-1) \stackrel{f}{\rightarrow} M \rightarrow M / f M \rightarrow 0$ provides an isomorphism

$$
\operatorname{Tor}_{i}^{S}(K, M / f M) \cong \operatorname{Tor}_{i}^{S}(K, M) \oplus \operatorname{Tor}_{i-1}^{S}(K, M)(-1)
$$


for all $i \in \mathbb{Z}$. By an easy induction argument this yields the isomorphisms in (a). The statement in (b) is well-known since $S / J_{r}$ has a linear resolution (see e.g. [3, Exercise A2.19]).

For a certain technical reason we need the following Lemma that describes the ideals $J_{\tilde{G}} \cap A_{n}$ respectively $J_{\tilde{G}} \cap B_{m}$ as binomial edge ideals.

Lemma 5.2. The ideal $J_{\tilde{G}} \cap A_{n}$ is the binomial edge ideal of the graph $G$ obtained by deleting all edges $\{i, j\}$ of the complete graph on $[n+m]$ vertices such that $n<i<j \leq m+n$. Similarly $J_{\tilde{G}} \cap B_{m}$ is the binomial edge ideal of the graph where all the edges $\{i, j\}$ of the complete graph on $[n+m]$ vertices such that $1 \leq i<j \leq n$ are deleted.

Proof. Let us consider the ideal $J_{\tilde{G}} \cap A_{n}$. Look at the primary decomposition of the graph $G$. We have to find all $\emptyset \neq T \subset[n+m]$ such that $c(T \backslash\{i\})<c(T)$ for all $i \in T$. If $T=\{1, \ldots, n\}$, then $c(T)=m>1$ and $c(T \backslash\{i\})=1$ for all $i$. Let $T \subset[n+m]$ denote a subset with $T \neq\{1, \ldots, n\}$. Then it is easy to see that the condition $c(T \backslash\{i\})<c(T)$ for all $i \in T$ can not be satisfied. So the claim follows by Lemma 2.2. A similar consideration proves the case of $J_{\tilde{G}} \cap B_{m}$.

As usual we define $\beta_{i, j}(M)=\operatorname{dim}_{K} \operatorname{Tor}_{i}^{S}(K, M)_{i+j}, i, j \in \mathbb{Z}$, the graded Betti numbers of $M$, a finitely generated $S$-module. Then $\operatorname{reg} M=\max \{j \in$ $\left.\mathbb{Z} \mid \beta_{i, j}(M) \neq 0\right\}$. In the following we shall prove that $S / J_{G}$ has a pure resolution. Note that all the $\beta_{i, j}\left(S / J_{G}\right)$ outside of the Betti table are zero.

Theorem 5.3. Let $S / J_{G}$ denote the binomial edge ideal of the complete bipartite graph $K_{m, n}$. Then the Betti diagram has the following form

\begin{tabular}{c|ccccc} 
& 0 & 1 & 2 & $\cdots$ & $p$ \\
\hline 0 & 1 & 0 & 0 & $\cdots$ & 0 \\
1 & 0 & $m n$ & 0 & $\cdots$ & 0 \\
2 & 0 & 0 & $\beta_{2,2}$ & $\cdots$ & $\beta_{p, 2}$
\end{tabular}

where

$$
p= \begin{cases}m, & \text { if } n=1 \\ 2 m+n-2, & \text { if } m \geq n>1 .\end{cases}
$$

Proof. Because of the regularity and depth of $S / J_{G}$, the non-vanishing part of the Betti table is concentrated in the frame of the one given in the statement. Clearly $\beta_{0,0}=1$ and $\beta_{i, 0}=0$ for all $i>0$. Furthermore $\beta_{1,0}=\beta_{2,0}=0$. Since $J_{G}$ is minimally generated by $m n$ binomials we get that $\beta_{1,1}=m n$ and $\beta_{1,2}=0$. 
In order to prove the statement we have to show that $\beta_{2,1}=0$ because this implies that $\beta_{i, 1}=0$ for all $i \geq 2$ as a consequence of the minimality of the free resolution. Here we have two cases:

Case(a): Let $m \geq n>1$. We take the short exact sequence (1) of Corollary 3.3. It induces a graded homomorphism of degree zero

$$
\operatorname{Tor}_{2}^{S}\left(K, S / J_{\tilde{G}}\right)=K^{b_{2}(m+n)}(-3) \rightarrow \operatorname{Tor}_{1}^{S}\left(K, S / J_{G}\right)=K^{m n}(-2) .
$$

Therefore it is the zero homomorphism. On the other side it induces a homomorphism

$$
\operatorname{Tor}_{3}^{S}\left(K, S / J_{\tilde{G}}\right)=K^{b_{3}(m+n)}(-4) \rightarrow \operatorname{Tor}_{2}^{S}\left(K, S / J_{G}\right),
$$

which is the zero homomorphism when restricted to degree 3 since $\operatorname{reg} S / J_{G}=$ 2 . Therefore there is a short exact sequence of $K$-vector spaces

$$
\begin{gathered}
0 \rightarrow \operatorname{Tor}_{2}^{S}\left(K, S / J_{G}\right)_{3} \rightarrow \operatorname{Tor}_{2}^{S}\left(K, S / J_{\tilde{G}} \cap A_{n}\right)_{3} \oplus \operatorname{Tor}_{2}^{S}\left(K, S / J_{\tilde{G}} \cap B_{m}\right)_{3} \rightarrow \\
\rightarrow K^{b_{2}(m+n)} \rightarrow 0 .
\end{gathered}
$$

That is $\beta_{2,1}\left(S / J_{G}\right)=\beta_{2,1}\left(S / J_{\tilde{G}} \cap A_{n}\right)+\beta_{2,1}\left(S / J_{\tilde{G}} \cap B_{m}\right)-b_{2}(m+n)$.

In the next step we shall compute $\beta_{2,1}\left(S / J_{\tilde{G}} \cap A_{n}\right)$ and $\beta_{2,1}\left(S / J_{\tilde{G}} \cap B_{m}\right)$. We start with the first of them. To this end we use the short exact sequence (2) of Corollary 3.3. At first we note that $\beta_{1,2}\left(S / J_{\tilde{G}} \cap A_{n}\right)=0$ which is true since $J_{\tilde{G}} \cap A_{n}$ is minimally generated by quadrics as follows by Lemma 5.2. Because of

$$
\beta_{3,0}\left(S / J_{\tilde{G}} \cap A_{n}\right)=\beta_{3,0}\left(S / J_{\tilde{G}}\right)=\beta_{2,1}\left(S / A_{n}\right)=0
$$

we get the following exact sequence of $K$-vector spaces.

$$
\begin{gathered}
0 \rightarrow \operatorname{Tor}_{3}^{S}\left(K, S / A_{n}\right)_{3} \rightarrow \operatorname{Tor}_{3}^{S}\left(K, S /\left(J_{\tilde{G}}, A_{n}\right)\right)_{3} \rightarrow \operatorname{Tor}_{2}^{S}\left(K, S / J_{\tilde{G}} \cap A_{n}\right)_{3} \rightarrow \\
\operatorname{Tor}_{2}^{S}\left(K, S / J_{\tilde{G}}\right)_{3} \rightarrow \operatorname{Tor}_{2}^{S}\left(K, S /\left(J_{\tilde{G}}, A_{n}\right)\right)_{3} \rightarrow 0 .
\end{gathered}
$$

By counting vector space dimensions this provides that

$$
\beta_{2,1}\left(S / J_{\tilde{G}} \cap A_{n}\right)=b_{2}(m+n)+\beta_{3,0}\left(S /\left(J_{\tilde{G}}, A_{n}\right)\right)-\beta_{2,1}\left(S /\left(J_{\tilde{G}}, A_{n}\right)\right)-\left(\begin{array}{c}
2 n \\
3
\end{array}\right) .
$$

Since $\left(J_{\tilde{G}}, A_{n}\right)=\left(J_{m}, A_{n}\right)$, where $J_{m}=I(n+1, \ldots, n+m)$ we might use Lemma 5.1 for the calculation of these dimensions. Therefore

$$
\begin{gathered}
\operatorname{Tor}_{3}^{S}\left(K, S /\left(J_{\tilde{G}}, A_{n}\right)\right)_{3} \cong \operatorname{Tor}_{0}^{S}\left(K, S / J_{m}\right)_{0}^{\left(\begin{array}{c}
2 n \\
3
\end{array}\right)} \text { and } \\
\operatorname{Tor}_{2}^{S}\left(K, S /\left(J_{\tilde{G}}, A_{n}\right)\right)_{3} \cong \operatorname{Tor}_{2}^{S}\left(K, S / J_{m}\right)_{3} \oplus \operatorname{Tor}_{1}^{S}\left(K, S / J_{m}\right)_{2}^{\left(\begin{array}{c}
2 n \\
1
\end{array}\right)}
\end{gathered}
$$


Therefore $\beta_{3,0}\left(S /\left(J_{\tilde{G}}, A_{n}\right)\right)=\left(\begin{array}{c}2 n \\ 3\end{array}\right)$ and $\beta_{2,1}\left(S /\left(J_{\tilde{G}}, A_{n}\right)\right)=b_{2}(m)+2 n b_{1}(m)$. Putting these integers together it follows that

$$
\beta_{2,1}\left(S / J_{\tilde{G}} \cap A_{n}\right)=b_{2}(m+n)-b_{2}(m)-2 n b_{1}(m) .
$$

Interchanging the rôles of $m$ and $n$ we derive a corresponding formula for $\left.\beta_{2,1}\left(S / J_{\tilde{G}} \cap B_{m}\right)\right)$, namely

$$
\beta_{2,1}\left(S / J_{\tilde{G}} \cap B_{m}\right)=b_{2}(m+n)-b_{2}(n)-2 m b_{1}(n)
$$

Finally we use both expressions in the above formula in order to confirm that $\beta_{2,1}\left(S / J_{G}\right)$ vanishes.

Case(b): Let $m>n=1$. Because $J_{\tilde{G}} \cap A_{1}=J_{G}$ we might use exact sequence (2) of Corollary 3.3. Since $\left(J_{\tilde{G}}, A_{1}\right)=\left(I(2, \ldots, n+m), A_{1}\right)$ the statements in Lemma 5.1 imply that $\operatorname{Tor}_{3}^{S}\left(K, S /\left(J_{\tilde{G}}, A_{1}\right)\right)_{3}=0$. Whence there is an exact sequence of $K$-vector spaces

$$
\begin{gathered}
0 \rightarrow \operatorname{Tor}_{2}^{S}\left(K, S / J_{G}\right)_{3} \rightarrow \operatorname{Tor}_{2}^{S}\left(K, S / J_{\tilde{G}}\right)_{3} \oplus \operatorname{Tor}_{2}^{S}\left(K, S / A_{1}\right)_{3} \rightarrow \\
\rightarrow \operatorname{Tor}_{2}^{S}\left(K, S /\left(J_{\tilde{G}}, A_{1}\right)\right)_{3} \rightarrow 0 .
\end{gathered}
$$

Therefore $\beta_{2,1}\left(S / J_{G}\right)=b_{2}(m+1)-\beta_{2,1}\left(S /\left(J_{\tilde{G}}, A_{1}\right)\right)$. Again by the statement of Lemma 5.1 (a) it follows that $\beta_{2,1}\left(S /\left(J_{\tilde{G}}, A_{1}\right)\right)=b_{2}(m)+2 b_{1}(m)$. Finally

$$
\beta_{2,1}\left(S / J_{G}\right)=b_{2}(m+1)-b_{2}(m)-2 b_{1}(m)=0,
$$

as required.

As a final feature of the investigations we will describe the explicit values of the Betti numbers $\beta_{2, i}\left(S / J_{G}\right), 2 \leq i \leq p$, as they are indicated in Theorem 5.3 .

Theorem 5.4. Let $G=K_{m, n}$ denote the complete bipartite graph with $m \geq$ $n \geq 1$.

(a) The Hilbert function of $S / J_{G}$ is given by

$$
\begin{gathered}
H\left(S / J_{G}, t\right)=\frac{1}{(1-t)^{m+n+1}}(1+(m+n-1) t)+\frac{1}{(1-t)^{2 m}}+\frac{1}{(1-t)^{2 n}} \\
-\frac{1}{(1-t)^{m+1}}(1+(m-1) t)-\frac{1}{(1-t)^{n+1}}(1+(n-1) t) .
\end{gathered}
$$

(b) For the multiplicity e $\left(S / J_{G}\right)$ it follows

$$
e\left(S / J_{G}\right)= \begin{cases}1, & \text { if } m>n+1 \text { or } n=1 \text { and } m>2, \\ 2 m, & \text { otherwise }\end{cases}
$$


(c) Let $n=1$. Then $\beta_{i, 2}\left(S / J_{G}\right)=m\left(\begin{array}{c}m \\ i\end{array}\right)-\left(\begin{array}{c}m \\ i+1\end{array}\right)-\left(\begin{array}{c}m+1 \\ i+1\end{array}\right)$ for all $2 \leq i \leq p=$ $m$. Let $m \geq n>1$. Then

$$
\begin{aligned}
\beta_{i, 2}\left(S / J_{G}\right)= & \left(\begin{array}{c}
m+n \\
i+2
\end{array}\right)+\left(\begin{array}{c}
2 n \\
i+2
\end{array}\right)+\left(\begin{array}{c}
2 m \\
i+2
\end{array}\right)+m\left(\begin{array}{c}
m+2 n-1 \\
i+1
\end{array}\right)+n\left(\begin{array}{c}
2 m+n-1 \\
i+1
\end{array}\right) \\
& -\left(\begin{array}{c}
m+2 n \\
i+2
\end{array}\right)-\left(\begin{array}{c}
2 m+n \\
i+2
\end{array}\right)-(m+n)\left(\begin{array}{c}
m+n-1 \\
i+1
\end{array}\right)
\end{aligned}
$$

for all $2 \leq i \leq p=2 m+n-2$.

Proof. In order to prove (a) we use the short exact sequences of Corollary 3.3. By the additivity of the Hilbert series we get the following equalities:

$$
\begin{aligned}
& H\left(S / J_{G}, t\right)=H\left(S / J_{\tilde{G}} \cap A_{n}, t\right)+H\left(S / J_{\tilde{G}} \cap B_{n}, t\right)-H\left(S / J_{\tilde{G}}, t\right), \\
& H\left(S / J_{\tilde{G}} \cap A_{n}, t\right)=H\left(S / J_{\tilde{G}}, t\right)+H\left(S / A_{n}, t\right)-H\left(S /\left(J_{\tilde{G}} A_{n}\right), t\right), \text { and } \\
& H\left(S / J_{\tilde{G}} \cap B_{m}, t\right)=H\left(S / J_{\tilde{G}}, t\right)+H\left(S / B_{m}, t\right)-H\left(S /\left(J_{\tilde{G}} B_{m}\right), t\right) .
\end{aligned}
$$

Substituting the Hilbert series of the complete graphs $S / J_{\tilde{G}}, S /\left(J_{\tilde{G}}, A_{n}\right)$ and $S /\left(J_{\tilde{G}}, B_{m}\right)$ as well as the Hilbert series of the polynomial rings $S / A_{n}, S / B_{m}$ we get the desired formula in (a). Then (b) is an easy consequence of (a).

For the proof of (c) we note at first the structure of the finite free resolution of $S / J_{G}$

$$
0 \rightarrow S^{\beta_{p}}(-p-2) \rightarrow \cdots \rightarrow S^{\beta_{3}}(-5) \rightarrow S^{\beta_{2}}(-4) \rightarrow S^{\beta_{1}}(-2) \rightarrow S
$$

with $\beta_{1}=m n \beta_{i}=\beta_{i, 2}\left(S / J_{G}\right), 2 \leq i \leq p$, as shown in Theorem 5.3. By the additivity of the Hilbert series this provides the following expression

$$
H\left(S / J_{G}, t\right)=\frac{1}{(1-t)^{2 m+2 n}}\left(1-\beta_{1} t^{2}+\sum_{i=2}^{p}(-1)^{i} \beta_{i} t^{i+2}\right)
$$

(see also [3, Exercise 19.14]). Now we use the expression of the Hilbert series $H\left(S / J_{G}, t\right)$ as shown in (a) and compare it with the one of the minimal free resolution. By some nasty calculations we derive the formulas for the Betti numbers as given in the statement.

Acknowledgement. The authors are grateful to Prof. Jürgen Herzog for drawing their attention to binomial edge ideals and suggesting their investigation.

\section{References}

[1] M. Brodmann, R. Sharp: Local Cohomology. An Algebraic Introduction with Geometric Applications. Cambr. Stud. in Advanced Math., No. 60. Cambridge University Press, (1998). 
[2] W. Bruns, J. Herzog: Cohen-Macaulay Rings, Cambridge University Press, 1993.

[3] D. Eisenbud: Commutative Algebra (with a View Toward Algebraic Geometry). Springer-Verlag, 1995.

[4] V. Ene, J. Herzog and T. Hibi: Cohen Macaulay Binomial edge ideals. Nagoya Math. J. 204 (2011) 57-68.

[5] S. Goto: Approximately Cohen-Macaulay Rings. J. Algebra 76, 214-225 (1982)

[6] J. Herzog, T. Hibi, F. Hreinsdotir, T. Kahle, J, Rauh: Binomial edge ideals and conditional independence statements. Adv. Appl. Math. 45 (2010) 317-333.

[7] H. Matsumura: Commutative Ring Theory, Cambridge Studies in Advanced Mathematics 8, Cambridge University Press, Cambridge (1986).

[8] P. Schenzel: On The Use of Local Cohomology in Algebra and Geometry. In: Six Lectures in Commutative Algebra, Proceed. Summer School on Commutative Algebra at Centre de Recerca Matemàtica, (Ed.: J. Elias, J. M. Giral, R. M. Miró-Roig, S. Zarzuela), Progr. Math. 166, pp. 241-292, Birkhäuser, 1998.

[9] P. Schenzel: On the dimension filtration and Cohen-Macaulay filtered modules. Van Oystaeyen, Freddy (ed.), Commutative algebra and algebraic geometry. Proceedings of the Ferrara meeting in honor of Mario Fiorentini on the occasion of his retirement, Ferrara, Italy. New York, NY: Marcel Dekker. Lect. Notes Pure Appl. Math. 206, 245-264 (1999).

[10] P. Schenzel: On birational Macaulayfications and Cohen-Macaulay canonical modules. J. Algebra 275 (2004), 751-770.

[11] R. P. Stanley: Combinatorics and commutative algebra. 2nd ed. Progress in Mathematics (Boston, Mass.). 41. Basel: Birkhuser (1996).

[12] R. H. Villarreal: Monomial Algebras, New York: Marcel Dekker Inc. (2001).

[13] S. ZAfAR: On approximately Cohen-Macaulay binomial edge ideal, Bull. Math. Soc. Sci. Math. Roumanie Tome 55(103) No. 4 (2012), 429-442. 
Peter SCHENZEL,

Martin-Luther-Universität Halle-Wittenberg,

Institut für Informatik,

D - 06099 Halle (Saale), Germany

Email: peter.schenzel@informatik.uni-halle.de

Sohail ZAFAR,

Department of Mathematics

University of Management and Technology,

Lahore, Pakistan

Email: sohailahmad04@gmail.com 
